\title{
los últimos progresos en perforación de minas, galerías Y canteras
}

$579-18$

G. VIÉ, ingeniero de minas

sinopsis Ha habido, durante estos últimos años, notables progresos en el campo de la perforación.

En este artículo se analizan algunos de ellos, tanto en el campo de maquinaria y herramientas, como en el de los explosivos.

Se ven los posibles campos de la perforación rotativa y la roto-percusión hídráulica, los usos del martillo ufondo del taladron, las características y posibles usos de los explosivos líquidos o pastosos; etc.

\section{NUEVAS MEJORAS en el CAMPo de la perforacion}

Los estudios sobre excavación de galerías y túneles siguen la evolur,ión de las ideas, y las realizaciones conseguidas desde el punto de vista de las máquinas son muy interesantes.

En la técnica más habitual (perforación de taladros para el derribo con explosivos) intervienen un cierto número de factores, cuya evolución nos permitirá encontrar una mejor soluc : desde el doble punto de vista del procedimiento y de la maquinaria de ejecución.

Hace unos 25 años, cuando las galerías eran de sección más reducida que ahora, parecía rias económico e incluso más rápido, debido a los altos rendimientos de los martillos perforadores disponibles, reducir el diámetro de los taladros a $30 \mathrm{~mm}$ y utilizar martillos perforadores de 25 a $30 \mathrm{~kg}$ sobre soporte neumático, abandonando entonces los supermartillos, jumbos y plataformas de perforación a los constructores de túneles.

Veinte años después asistimos a la evolución inversa, debido a múltiples razones, entre las que se encuentran el aumento de las secciones, con lo que se modifica: las condiciones de trabajo del explosivo, las posibilidades de evolución de las máquinas, la importancia de cada operación elemental, así como el perfeccicnamiento de las instalaciones, aumento de la potencia de los martillos perforadores y de la manejabilidad de los jumbos...

Este tipo cíclico de evolución se manifiesta en diferentes puntos; por ejemplo se está viendo en las barrenas que la relación entre la calidad de las barras y la de las bocas de las barrenas hace inclinar la balanza, bien a favor del uso de bocas recambiables, o bien a favor de las barrenas de una sola pieza.

La dificultad de poder utilizar una plataforma de carga y un jumbo de perforación en una obra de sección pequeña, en donde la operación de perforar tiene una importancia más bien pequeña, no fue el único motivo de que no se empleara esta maquinaria poco rentable, pues a la gran superficie ocupada por los jumbos se añadía la dificultad de su manejo.

Estos jumbos antiguos no tienen comparación posible con los actuales, accionados hidráulicamente y mucho más "concentrados" en sus piezas esenciales. La eliminación del jumbo, por este motivo, establecía la supremacía de los martillos perforadores de peso medio, sobre soporte neumático, eliminando no sólo los martillos perforadores pesados, sino también los equipos vibro-rotativos, limitando el uso de las perforadoras rotativas a la perforación de las rocas blandas.

El gran número de obras abiertas durante los dos o tres últimos decenios, la confrontación de los medios, y de sus resultados, incitaban a la elaboración de una nueva doctrina.

$Y$ en este momento parece que nos encontramos ante un verdadero vuelco de las tendencias. 


\section{CAMPOS RESPECTIVOS DE LA PERFORACION ROTATIVA Y LA ROTO-PERCUSION}

La experiencia demuestra que cuando la perforación rotativa puede emplearse se obtienen mejores resultados, y costos menos elevados.

Sin embargo, subsiste la dificultad de elegir entre la perforación rotativa y roto-percusión hidráulica.

Una firma francesa (Secoma) obtuvo un gráfico que permite hacer mejor esa elección, después de la realización de ensayos sobre muestras de roca para conocer sus características principales: perforabilidad y abrasividad, esta última función de la dispersión o concentración de sílice en la susodicha roca.

Estos son, esencialmente, los dos parámetros que permitieron la obtención del diagrama dividido en cuatro zonas:

\section{Zona 1}

Zona de dureza muy débil y de poca abrasividad, dominio de la perforación rotativa en seco, presión pequeña.

\section{Zona 2}

Zona de débil dureza y poca abrasividad, dominio de la perforación rotativa en seco, o con inyección de aire a presión media.

\section{Zona 3}

Zona de dureza media y poca abrasividad, dominio de la perforación rotativa, empuje a gran presión con inyección de agua a alta presión. El empuje sobre la barrena puede llegar hasta $2.000 \mathrm{~kg}$.

\section{Zona 4}

Zona de gran dureza y mucha abrasividad. Dominio de la roto-percusión hidráulica.

Es evidente que las zonas 1,2 y 3 son perforables con roto-percusión hidráulica, sin embargo, en ese caso, este tipo de perforación ni dará muy buenos resultados (a veces incluso muy débiles: fenómeno de relleno), ni una perforación económica (el golpe fatiga mucho más las herramientas que una simple rotación).

Por regla general, cuando las rocas son muy duras para poder realizar la perforación simplemente rotativa, se recurre a la perforación vibro-rotativa con presiones muy grandes sobre las herramientas.

Se han efectuado ensayos comparativos en casi todos los países, pero especialmente en Alemania y Francia, entre aparatos vibro-rotativos y martillos potentes, tradicionales, de hasta un peso de $72 \mathrm{~kg}$. En los ensayos Friedrich Heinrich, los progresos fueron del mismo orden.

Podemos decir a favor de la perforación vibro-rotativa, que hay una disminución del nivel sonoro, de 9 a 11 decibelios menos. Pero la misma ventaja se encuentra en la perforación por percusión con el martillo hidráulico.

Según los ensayos, parece ser que el promedio de vida de las bocas se multiplica por 4 y la de las barras, por 3.

La perforación vibro-rotativa es mirada también con interés en Gran Bretaña en su solución totalmente hidráulica, y aunque todavía no está perfeccionada parece que existe un gran porvenir para este método. Para fijar ideas y orientar quizás las investigaciones, la perforación hidráulica rotativa con velocidad de rotación y presiones variables, en rocas poco abrasivas, debería alcanzar velocidades de 1,5 a $3,5 \mathrm{~m} / \mathrm{min}$, y la perforación hidráulica por percusión, para rocas muy difíciles de perforar, velocidades del orden 1,4 a $2,3 \mathrm{~m} / \mathrm{min}$.

\section{PERFORACION POR PERCUSION}

La utilización del fluido hidráulico para la transmisión de potencia se ha generalizado. Reproducimos dos fotografías de una máquina reciente (Hydrofore), un motor Diesel con un grupo de 


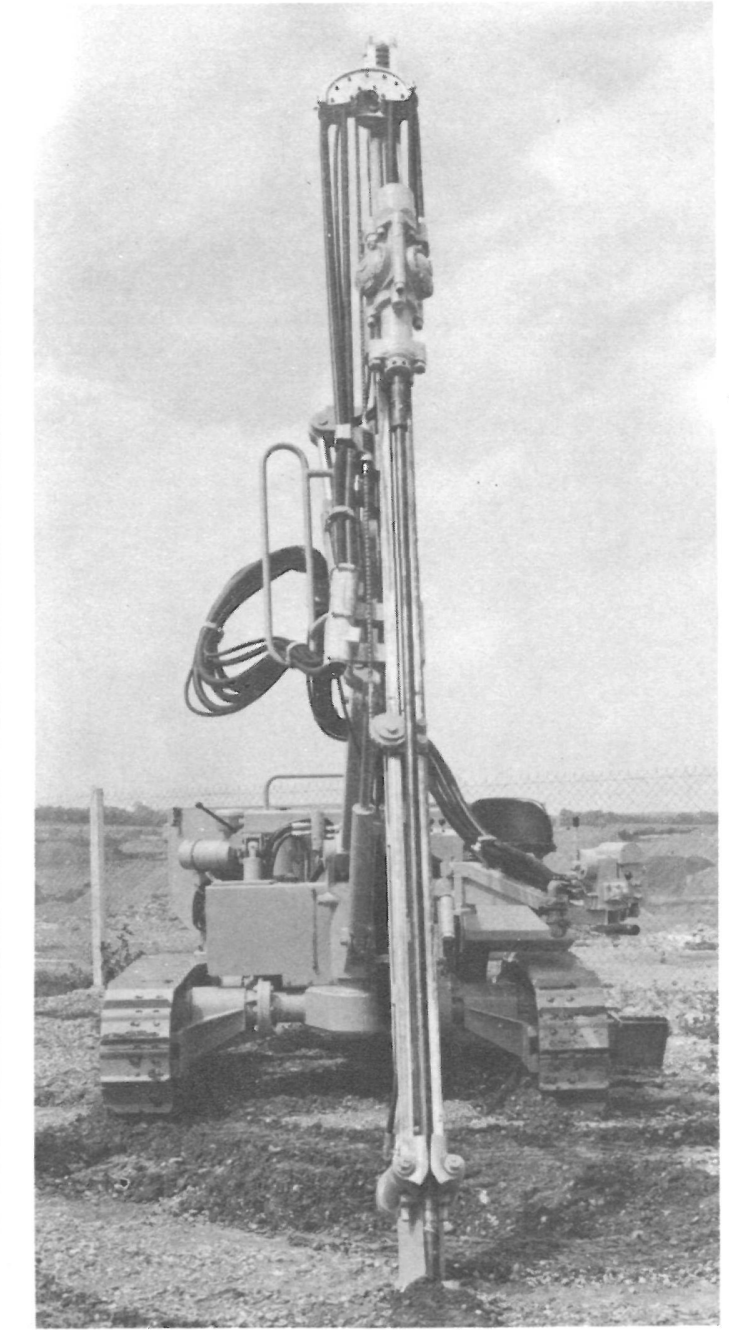

bombeo que alimenta todas las funciones hidráulicas, lo que permite una reducción apreciable del precio de coste del metro perforado. La potencia máxima absorbida por el instrumento de perforación, y su remolcador, es del orden de $40 \mathrm{CV}$, para el modelo sobre neumáticos, y de $50 \mathrm{CV}$, para el modelo sobre orugas.

Para las perforaciones con inyección de aire se añade un compresor de 50 a $60 \mathrm{CV}$. Con una potencia total de 90 a $110 \mathrm{CV}$, esta combinación permite avances comparables a los de los aparatos exclusivamente "con aire comprimido" de una potencia total de 200 CV o más.

Abriremos un paréntesis a propósito de las herramientas empleadas en los extremos del varillaje.

En perforación por percusión, el progreso se manifiesta en los detalles considerados como accesorios.

Eso ocurre por ejemplo en bocas cuyo papel es considerado, sin embargo, esencial.

Recientemente, ha aparecido una nueva gama de bocas cuya cara puesta en contacto directo con la roca (y que soporta el efecto de los choques sobre esta última) está provista de "botones" de carburo de tungsteno, en sustitución de las plaquitas en cruz que habían constituido ya un progreso cierto.

La idea se inspira, más o menos, en la disposición adoptada en algunos trépanos triconos, cuya parte dentada estaba provista de esas "pastillas" o "botones" para penetrar en rocas duras.

Estas nuevas bocas con botones de carburo, y diámetro comprendido entre 51 y $102 \mathrm{~mm}$, nos dan un rendimiento muy superior a las bocas con plaquitas cualquiera que sea la naturaleza de la roca:

- El intervalo entre dos reafilados dura de 4 a 5 veces más.

- El grado de penetración es sensiblemente mayor (10 a $20 \%$ ).

- La perforación se realiza con más suavidad y uniformidad, debido a los numerosos puntos de contacto con el fondo del taladro. 




Tipo de cabeza de diámetro 50 a $102 \mathrm{~mm}$, con botones de carburo de tungsteno para perforaciones destinadas al arranque en gran masa de rocas, por gradas.

SANDVIK ya había comercializado antes las bocas de botones, pero esta última gama es de una concepción totalmente nueva por su perfil, por las características del cuerpo de la boca, por la calidad del carburo de los botones y, finalmente, por la técnica de engarce de estos últimos.

Estas bocas tienen una aplicación muy rentable en la perforación de orificios relativamente profundos, en los que la existencia de intervalos grandes entre reafilados proporciona una economía muy sustanciosa. Estos reafilados que consisten, de hecho, en simples rectificaciones pueden hacerse en la misma obra con una amoladora neumática de mano.

El coste de reafilado por metro perforado es 4 veces menor en las bocas de botones, que en las bocas convencionales. Se estima que es necesario reafilar 12 a 15 veces una boca normal a lo largo de su vida, mientras que para las bocas de botones basta con rectificarlas 4 a 5 veces durante toda su vida.

Un aspecto muy importante, y que normalmente pasamos por alto, es el de la "calidad" o "regularidad" del taladro obtenido.

Una perforación muy cilíndrica, convenientemente "calibrada", es ventajosa desde el punto de vista de la carga del explosivo.

Esta regularidad de calibre influirá en el rendimiento del tiro (voladura), ya que es conveniente reducir, tanto como sea posible, la diferencia entre el diámetro del taladro y el del cartucho del explosivo. En principio, la relación óptima es de 1,2 a 1,25.

El aparato de perforación, y la herramienta fijada al extremo de las barras o varillaje de perforación, deben escogerse con mucho cuidado, teniendo muy en cuenta el precio de coste en metro perforado.

También es conveniente cambiar el tipo de boca en función del terreno que se va a atravesar.

La evolución hacia la perforación de taladros de $48 \mathrm{~mm}$ de diámetro (cartuchos de $\varnothing 40$ ) con martillos de gran potencia, lleva al aumento de la sección de las barrenas.

Las barrenas hexagonales de $22 \mathrm{~mm}$ se reservan para los martillos de 25 a $40 \mathrm{~kg}$, con díametros de los taladros de $42 \mathrm{~mm}$, pero en seguida se pasa a las hexagonales lísas de $25 \mathrm{~mm}, 0$ a las redondas de $32 \mathrm{~mm}$ e, incluso, a diámetros superiores en las barrenas con varias piezas.

Respecto al número de piezas de que se compone una barrena la elección no está clara; se dan todas las soluciones, desde la barrena de una sola pieza hasta las barrenas con 3 ó 4 elementos: boca recambiable, barra, enmangadura recambiable de una o dos piezas.

66 
Un problema que aún no está completamente resuelto es el del ajuste de las partes recambiables, bien a rosca o por mango cónico, que permiten realizar las prolongaciones de las barrenas. En todas las prolongaciones existe siempre una pérdida de potencia de golpe.

Cada vez más las herramientas de perforación, y de los equipos de carga de los escombros de los taladros, reciben la energía necesaria para su funcionamiento, por medio de bombas, o "centrales" hidráulicas cuyo fluido está formado por aceite a presión variable.

La bomba, o la central hidráulica, se alimentan de la energía de un motor Diesel, o de un motor eléctrico.

O sea, en vez de aire comprimido a $70,100,120$ ó $170 \mathrm{~N} / \mathrm{cm}^{2}$, el fluido de transmisión de energía es aceite a $1.000 \mathrm{~N} / \mathrm{cm}^{2}$.

Esta técnica hidráulica permite rendimientos energéticos del orden del 80 al $90 \%$, mientras que el aire comprimido es un pésimo agente de transmisión. Incluso el golpe de una herramienta se realiza por hidráulica con lo que se consiguen mayores avances y mayor ahorro de fuerza.

La atmósfera de las obras subterráneas no es contaminada ya por los escapes tan desagradables de los motores Diesel que trabajan en ambientes cerrados, a pesar de los sistemas de depuración de gases tóxicos de que están provistos estos motores de combustión.

Uno de los ejemplos más significativos y recientes de materiales ultramodernos empleados en galerías es la combinación de perforadoras, con palas cargadoras de dientes y de vagones de transporte sobre raíles o "Shuttletrain" para evacuar el escombro. Este conjunto es de fabricación sueca.

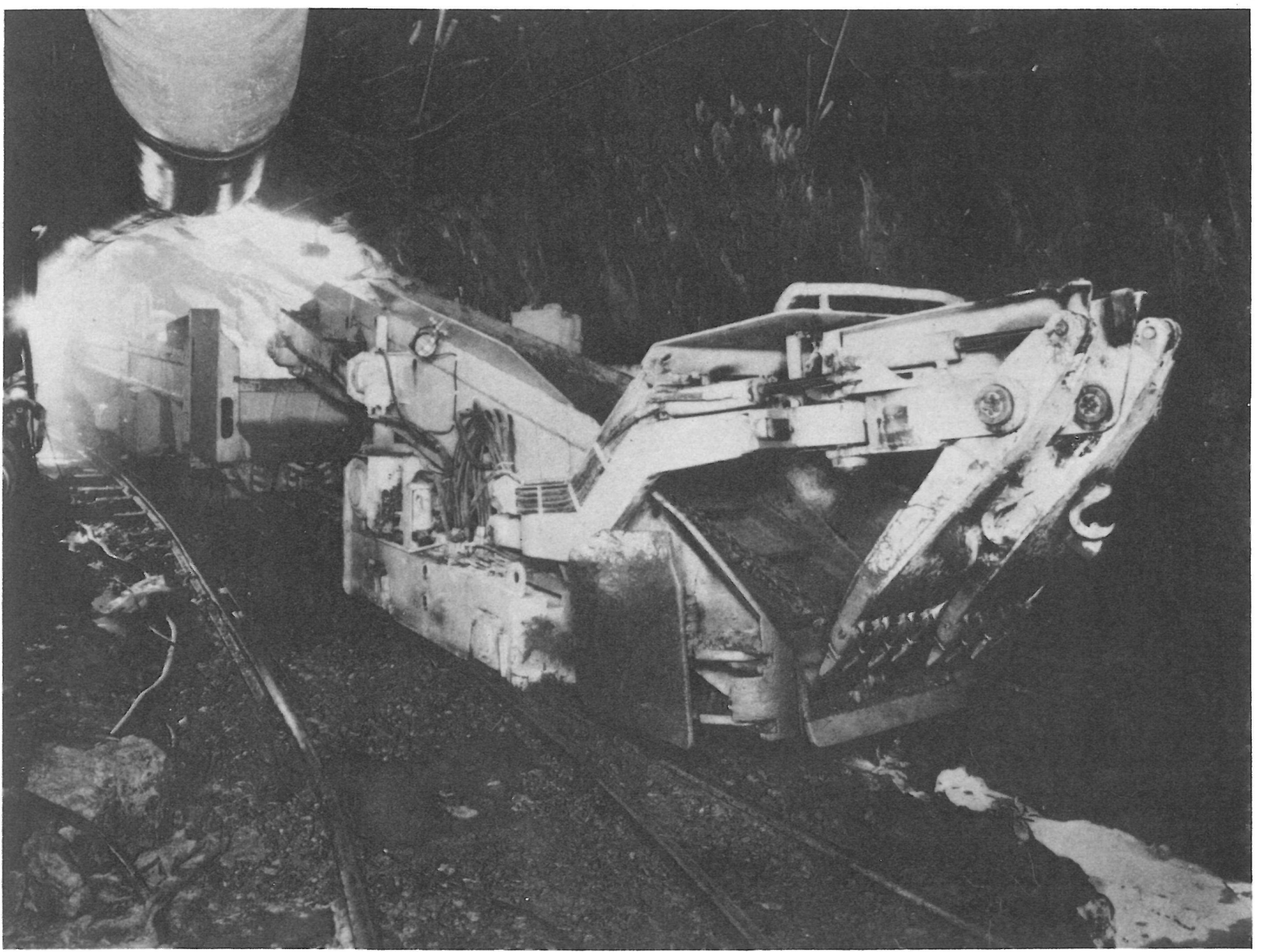

Haggloader y Shuttletrain (material sueco Hagglund) en servicio en la perforación del túnel Bolmen (Suecia). 
Este equipo se ha usado en la excavación del Túnel de Bolmen, una obra de gran importancia realizada desde el lago de Bolmen (provincia de Smaland), hasta Perstrop (provincia de Skane), con una sección de $7,5 \mathrm{~m}^{2}$, con miras al abastecimiento de agua potable de toda una región.

La particularidad de estas máquinas es que funcionan por electricidad como energía primaria con lo que se eliminan por completo el polvo y la contaminación.

\section{PERFORAGION POR MARTILLO EN EL FONDO DEL TALADRO (Down the Hole)}

En el número 55 de Informes, aparecido en el año 1953, publicamos un artículo con el título de "ARRANQUE EN CANTERAS CON TIROS VERTICALES PROFUNDOS", en el que se describe la técnica, entonces completamente nueva, del empleo del "Martillo en el fondo del taladro".

Poco después de la aparición de este artículo, algunas fábricas de cemento españolas, adquirieron la máquina Stenuick, para emplear este procedimiento.

En los primeros años, se le podía reprochar ser demasiado lenta, ya que el desplazamiento no pasaba casi nunca de los $3 \mathrm{~m} / \mathrm{h}$. El diámetro del pistón estaba limitado por el del orificio que se había de perforar, lo que producía una limitación de la energía de choque de cada golpe sobre el fondo del taladro.

Para paliar este inconveniente se podía alargar el recorrido del pistón, pero entonces había otro problema, pues se alargaba la duración del trayecto de ida y vuelta y, por lo tanto, se disminuía la frecuencia del golpe.

Conscientes de este inconveniente, los constructores intentaron solucionarlo mediante la "sobrealimentación" de los martillos "fondo del taladro" con aire comprimido a presión más elevada: 100,120 y hasta $170 \mathrm{~N} / \mathrm{cm}^{2}$.

Es indudable que con una mayor presión, el martillo "fondo del taladro" llega e incluso sobrepasa a la potencia de golpe del martillo clásico; de esta forma puede luchar con ventaja frente a él en lo que se refiere a la rapidez de perforación, manteniendo siempre la supremacía en los terrenos difíciles. Al mismo tiempo, el escape de aire del martillo cuyo rendimiento aumenta sirve para realizar una gran parte del soplado, pudiendo incluso llegar a la totalidad.

En algunas rocas abrasivas la boca se desgasta rápidamente y el diámetro del taladro disminuye sensiblemente a medida que aumenta su profundidad.
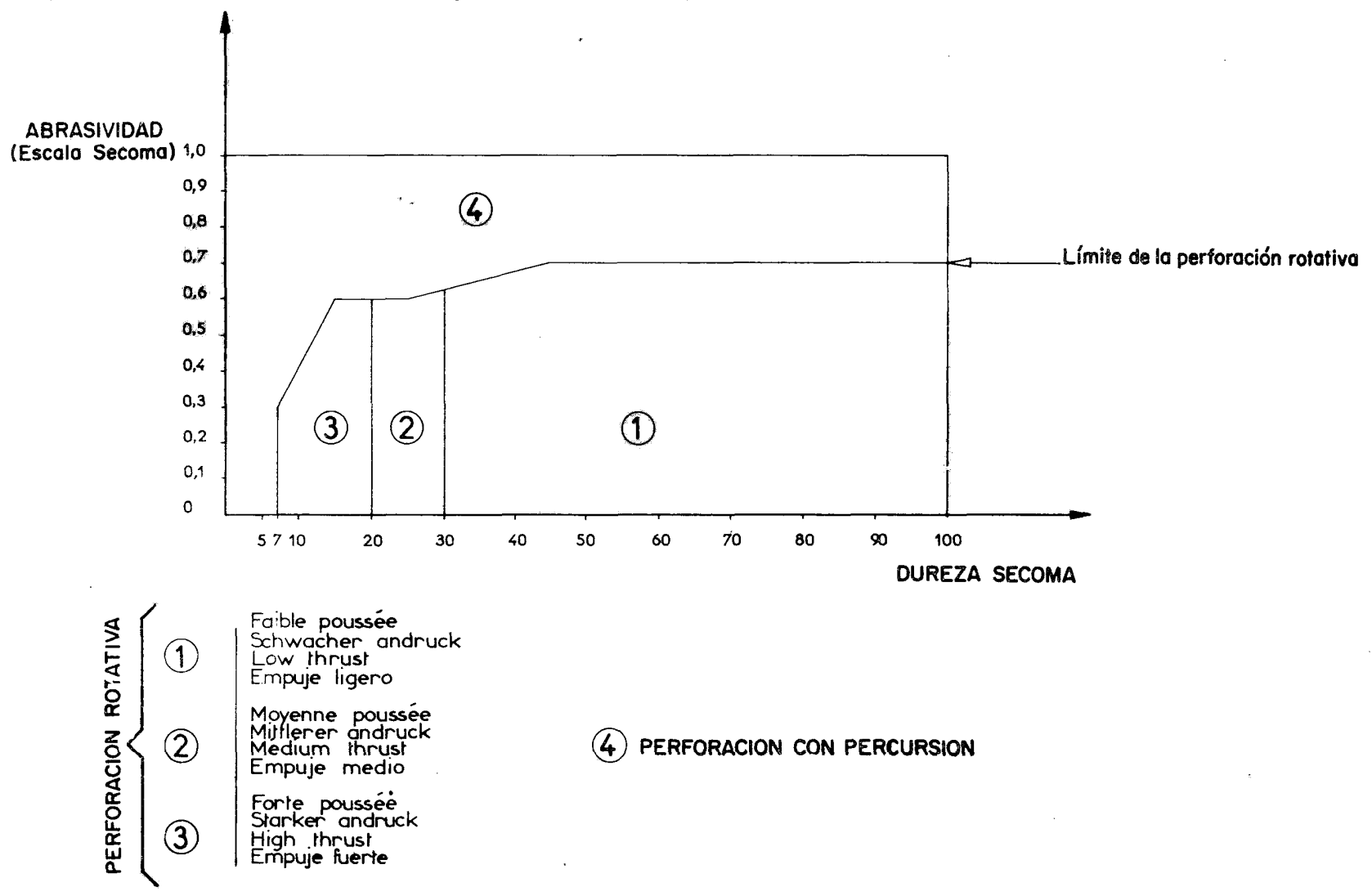

(4) PERFORACION CON PERCURSION 
Eso puede limitar el uso del martillo "fondo del taladro" cuyo diámetro debe, evidentemente, quedar netamente inferior al del orificio perforado.

Para diámetros de $100 \mathrm{~mm}$ y mayores, ofrece ventajas el martillo "fondo del taladro", y su comportamiento es superior en la perforación de terrenos accidentados. El soplado es enérgico y se puede perforar a gran profundidad.

También podríamos citar como ventajas:

- Gran velocidad de perforación siempre que se use aire comprimido a alta presión (100 $\mathrm{N} / \mathrm{cm}^{2}$ o más).

- Ahorro debido a que el tren de tubos es mucho más barato que el varillaje usado hasta ahora para los alargamientos de las barrenas.

- Ahorro sensible de aire, compensado, sin embargo, por el hecho de que la energía a alta presión es mucho más cara que la energía a presión normal.

- Funcionamiento más silencioso, ya que el martillo trabaja en el fondo del taladro.

La experiencia demuestra, una vez perfeccionado este equipo, que se perfora prácticamente dos veces más rápido con una presión de aire de $180 \mathrm{~N} / \mathrm{cm}^{2}$ que con una de $105 \mathrm{~N} / \mathrm{cm}^{2}$, siendo el comportamiento de la boca prácticamente el mismo.

Con $180 \mathrm{~N} / \mathrm{cm}^{2}$ de presión se puede obtener una velocidad instantánea de 40 a $45 \mathrm{~m} / \mathrm{h}$ ẹn un terreno calcáreo duro.

En terrenos duros, la toma de muestras por perforación no puede hacerse más que por rotación con coronas de diamantes girando a $2,5 \mathrm{~m} / \mathrm{seg}$. Ahora bien, con martillo en el fondo del taladro, la recuperación de los detritus permite obtener datos, a menudo suficientes, sobre la naturaleza de las capas atravesadas.

En obras públicas las aplicaciones de este tipo de martillo son múltiples, por ejemplo:

- Realización de perforaciones para la colocación de tirantes de anclaje, pantallas de inyección y de drenaje.

- Perforaciones en terrenos de aluvión por el método de vibro-percusión.

- Realización de perforaciones en agua, lo que prueba que la presencia de agua no está reñida, teniendo ciertas precauciones, con el empleo del martillo "fondo del taladro". Se han hecho perforaciones en agua hasta una profundidad de $150 \mathrm{~m}$, con $\varnothing 250 \mathrm{~mm}$, en una caliza dura y fisurada.

En los trabajos de cimentación del oleoducto petrolífero de Alaska, un martillo de este tipo consiguió perforar en una sola pasada orificios de $750 \mathrm{~mm}$ de diámetro en el terreno permanentemente helado, hasta una profundidad de $20 \mathrm{~m}$, siendo alimentado por un compresor de $600 \mathrm{CV}$.

Por último, para subrayar aún más la importancia de los progresos hechos en este terreno, añadiremos que la técnica de la perforación con martillo "fondo del taladro" se ha usado en la investigación y explotación petrolífera, hasta $4.000 \mathrm{~m}$ por debajo de la superficie, con diámetros que van desde 3 hasta 30 pulgadas.

\section{EVOLUCION DE LOS EXPLOSIVOS}

Una exposición de los progresos en materia de perforación de minas quedaría incompleto sin un capítulo relativo a los explosivos de reciente elaboración.

Después de la pólvora negra, la dinamita, y los explosivos nitrados o cloratados, se ha llegado al nitrato-fuel que puede meterse a granel dentro de una perforación.

La mezcla AN + FO, aunque es a la vez barata y de fácil aplicación, desgraciadamente libera poca energía.

Para disponer de una potencia superior, conseguir un comportamiento mejor dentro del agua y, a la vez, aumentar la seguridad, se idearon los "slurries" o "bouillies" (lechadas o pastas), 
auténticos explosivos líquidos, que se están intentando usar en las perforaciones de pequeño y mediano diámetro (inferiores a $120 \mathrm{~mm}$ ).

Las fórmulas son complicadas y están siendo perfeccionadas desde hace 20 años en que 'hicieron su aparición.

La primera generación de "slurries" tenía como materia primera un explosivo; por ejemplo: nitroglicerina, pólvoras sin humo, tolita, pentolita, exolita.

Curiosamente, en la segunda generación, hacia el año 1966, había sólo ingredientes no explosivos: nitratos minerales u orgánicos, polvo de aluminio y aditivos diversos.

Esa segunda generación sería sustituida más tarde por una tercera, formada por un simple gel de nitrato-fuel 0 de otras mezclas combustible + comburente.

Para citar un ejemplo más detallado de uno de estos compuestos examinaremos a continuación los "bouillies" IRECO, a base de nitratos minerales:

1. Una solución de nitrato amónico, sódico o, a veces, cálcico, con un aditivo de goma natural o artificial que cristaliza hacia los $32^{\circ} \mathrm{C}$. Esta solución debe conservarse por encima de los $45^{\circ} \mathrm{C}$, pero por debajo de los $60^{\circ} \mathrm{C}$ para evitar la degradación de la goma.

Esta solución representa el $60 \%$ del compuesto final.

2. Un nitrato amónico seco, entre un 17 ó un $27 \%$, según sean las combinaciones químicas.

3. Una mezcla (o Premix) de polvo de aluminio, de azufre, y de gilsonite (betún muy sulfuroso), en la proporción del 3 al $14 \%$.

4. Un agente reticulante a base de bicromato sódico $(0,1 \%)$ para hacer variar la consistencia de la mezcla.

5. Un aligerante $(0,2$ a $0,8 \%)$ formado por una solución de nitrato sódico, que al desprender burbujas de nitrógeno, permite variar la densidad y a la vez, eventualmente, la sensibilidad de la mezcla explosiva.

La rapidez de estas dos últimas reacciones es casi inmediata (unos minutos como máximo) . Entre las ventajas de este compuesto están la de que es poco sensible a la inflamabilidad, su sensibilidad al choque es 20 veces menor que la de la dinamita, su sensibilidad al rozamiento es 3 veces menor, y de ahí sus mejores coeficientes de seguridad.

La presencia del agua en las perforaciones que se introducen estos compuestos provoca una destrucción no demasiado progresiva, pero que termina siendo total después de un tiempo (bastante largo) de contacto.

Además, la presencia del agua en la fórmula de estos compuestos facilita la descomposición total en el momento de la deflagración, y los humos tóxicos se reducen, con lo que no se altera la atmósfera de la galería o del túnel.

Desde el punto de vista de la potencia, esta última es comparable a la de una goma dinamita con el $30 \%$ de nitroglicerina, con una velocidad de detonación comprendida entre $3.700 \mathrm{~m} / \mathrm{seg}$ y $4.300 \mathrm{~m} / \mathrm{seg}$, según sea la composición.

Otra de las ventajas de una mezcla explosiva líquida, o siruposa bombeable, es que su consistencia puede corregirse o m dificarse según cuáles sean las necesidades $y$, por tanto, su densidad es susceptible de pasar de 0,9 a 1,20 , o a la inversa.

No hay nada nuevo respecto a la carga de los taladros mediante explosivos de cartucho, ni respecto a su introducción a granel por simple gravedad (caso del nitrato-fuel).

Sin embargo, en los taladros horizontales, los explosivos granulados pueden colocarse mediante aire comprimido (caso de las minas de hierro, por ejemplo), mientras que los "slurries" pueden serlo por bombeo.

En las perforaciones verticales, o ligeramente inclinadas, siempre que su diámetro sea suficiente, el relleno se realiza fácilmente por gravedad, cuando se trata de pastas líquidas o siruposas.

Un caso cada vez más frecuente es el de las excavaciones en masa, en canteras a cielo abierto. 
De la misma forma que la carga gravitatoria a granel, el vertido de explosivos líquidos, bien por bombeo o no, favorece el rendimiento de un tiro, ya que el taladro de esta manera queda completamente lleno por el explosivo, abarcando también los bordes irregulares del taladro.

Se realiza, de esta manera, una concentración del explosivo, lo que no cabría esperar de los explosivos de cartucho.

De todas formas, el colchón de aire entre el cartucho y las paredes del orificio desaparece.

Es fácil admitir que el contacto se favorece más con un explosivo líquido, incluso pastoso, y que la mejora del rendimiento de una voladura sea del 15 al $25 \%$ según los casos.

Las pastas explosivas "slurries" pueden transportarse en camiones-cisterna especiales, hasta pie de obra, en donde se efectuará la carga de una voladura (si vienen prefabricadas) o se fabricarán en el sitio mismo de empleo en el momento de su vertido.

Este problema ya está solucionado para los taladros verticales en cantera, de un diámetro superior a $5 \mathrm{~mm}$. Pero el bombeo en taladros horizontales de pequeño diámetro está en estudio, y no hay duda que se resolverá.

Independientemente de un mejor rendimiento, el bombeo de explosivos líquidos, o al menos "siruposos" significa un ahorro de tiempo.

Una unidad de camión-bomba que sirve a la vez para la fabricación y la carga de un tiro (voladura) permite la colocación en taladros de $105 \mathrm{~mm}$, de $5 \mathrm{t}$ de "slurries", con 3 hombres en 3 horas, mientras que el método tradicional nos llevaría el doble de tiempo.

Una última nota respecto al rendimiento del explosivo:

La distancia de proyección de las rocas en una voladura es, sobre todo, función del consumo específico de explosivo por $\mathrm{m}^{3}$ de roca in situ.

Este consumo puede reducirse disminuyendo el número de taladros y aumentando su profundidad.

El aumento de granulometría en las rocas fragmentadas no tiene porqué afectar necesariame ce al rendimiento de las máquinas de recogida y carga.

Una buena medida de precaución, según se avanza por la galería, es hacer los taladros de la boca a una altura mínima respecto al suelo de la obra, y prever un retraso de 50 milisegundos entre la voladura de estos taladros y la de los otros llamados de gálibo.

\section{PERFORADORES CONTINUOS (TOPOS) EN GALERIAS DE ROCAS DURAS}

Una máquina Wirth había trabajado anteriormente en el granito de Mont-Blanc, perforando un pozo inclinado de $1.150 \mathrm{~m}$ y $3 \mathrm{~m}$ de $\varnothing$ en condiciones satisfactorias.

Un perforador continuo del mismo constructor alemán fue seleccionado para perforar la galería de l'Echaillon, cerca de Saint Jean de Maurienne, actuando sobre conglomerados esquistos y gres con $\varnothing$ de $5,80 \mathrm{~m}$ y $4.700 \mathrm{~m}$ de longitud, alcanzando penetraciones de hasta $30 \mathrm{~m}$ diarios.

Siempre por encargo de d'ELECTRICITE de FRANCE, I'Aménagement Arc-Isère ha conseguido ocasiones nuevas de experimentar los perforadores continuos como la galería del Grand Chatelard con $6.080 \mathrm{~m}$ de longitud y $6,80 \mathrm{~m} \varnothing$ en sección total y $5,80 \mathrm{~m} \varnothing$ con revestimiento. La galería bajo el macizo de Belledonne, con un $\varnothing$ de $5,80 \mathrm{~m}$ y en lugares aislados de $5,30 \mathrm{~m}$ y $5,10 \mathrm{~m} \varnothing$ según la naturaleza del revestimiento y una longitud total de $18.200 \mathrm{~m}$. La galería Brame Farine, de $3.920 \mathrm{~m}$ de longitud y $8,10 \mathrm{~m} \varnothing$, lo que se traducía en $7,50 \mathrm{~m}$ a $7,30 \mathrm{~m} \varnothing$ de las secciones revestidas, encontrándose muy malos terrenos, por lo que el ataque tuvo que realizarse con un perforador continuo Robbins. De todas formas, la perforación percutante o de golpeo tradicional se ha utilizado en algunos puntos para la perforación que se ejecutó en su mayor parte con perforadores continuos.

El perforador continuo Robbins, con su cinta continua de evacuación de escombros y sus instalaciones de ventilación y antipolvo, forman un conjunto de $70 \mathrm{~m}$ de longitud.

La cabeza, susceptible de perforar en sección bruta $8,10 \mathrm{~m} \varnothing$, está movida por seis motores de $150 \mathrm{CV}$ cada uno y lleva 51 muelas de un peso unitario de 50 a $60 \mathrm{~kg}$. 
Los elementos de corte están colocados en doce cangilones que evacuan a través de un pasillo que atraviesa el centro de la máquina hacia un tren de cinco vagonetas de $15 \mathrm{~m}^{3}$ cada una. El convoy que se forma es propulsado hacia el exterior por un tractor Diesel de $160 \mathrm{CV}$ y conducido con rayo Láser en su conjunto (generador Spectra Physics).

El perforador continuo Witrh TBV $580 \mathrm{H}$ que se empleó en las galerías antes mencionadas mide un total de 28,6 m y está equipado, esencialmente, por una viga de $7,2 \mathrm{~m}$ de longitud de sección cuadrada de 1,4 m de lado, empujada por cuatro gatos que desarrollan un empuje de hasta 700 t. Lleva en la parte de atrás seis motores hidráulicos afectados por la rotación de la cabeza de perforación de $5,88 \mathrm{~m} \varnothing$ con 38 muelas que cada una soporta empujes de 8,5 t.

Conducción topográfica por Láser (generador Spectra Physics $5 \mathrm{~mW}$ ). La perforación ha alcanzado gneis, esquistos cristalinos, esquistos negros y conglomerados carboníferos.

En los gneis, donde el avance horario ha variado con esta máquina Wirth entre $25 \mathrm{~cm}$ y $1 \mathrm{~m}$ por hora, la velocidad de rotación era de seis vueltas por minuto. Se ha experimentado un avance por vuelta (profundidad de paso) del orden de 1 a $2 \mathrm{~mm}$, según el estado de uso de las muelas.

\section{résumé}

Derniers progrès en foration de mines, galeries et carrières

G. Vié, ingénieur des mines

Des progrès notables ont eu lieu, ces dernières années, dans le domaine de la foration.

Cet article en analyse quelques-uns tant du domaine des engins et outils que de celui des explosifs.

On y commente les domaines possibles de la foration rotative et roto-percutante hydraulique, les emplois du marteau ufond de troun, les caractéristiques et usages possibles des explosifs liquides et pâteux, etc.

\section{summary}

Recent progress in drilling and excavation in mines, tunnels and quarries

G. Vié, Mining engineer

Important progress has been achieved in recenht years inh the field of drilling and excavation. This article studies some of these improved methods, both in the area of machines and equipment and the explosives field.

The potential areas of application of rotary drilling and hydraulic rotary-percussion drilling, the uses for the uborehole bottoms hammer, the properties of powder and liquid explosives and their possible applications, etc., are examined.

\section{zusammenfassung}

Die letzten Fortschrítte in der Bohrung von Bergwerken, Schaechten und Steinbruechen

G. Vié, Bergbauingenieur

In den letzten Jahren wurden beachtliche Fortschritte auf dem Gebiet der Bohrungen erzielt.

In diesem Artikel werden verschiedene dieser Fortschritte untersucht, sowohl was das gebiet der Maschinen uls

Es werden die möglichen Gebiete der Rotationsbohrunge und der hydraulischen RotoPerkussion, die Gebrauchsmöglichkeiten Hammers für den "Grund der Bohrung", die Merkmale und möglichen Anwendungen der flüssigen und pastigen Sprengstoffe, usw. beschrieben. 